\title{
NONCROSSING NORMAL ORDERING FOR FUNCTIONS OF BOSON OPERATORS
}

\author{
TOUFIK MANSOUR, MATTHIAS SCHORK AND SIMONE SEVERINI
}

Department of Mathematics, University of Haifa, Haifa 31905, Israel

Alexanderstrasse 76, 60489 Frankfurt, Germany

Institute for Quantum Computing and Department of Combinatorics and Optimization, University of Waterloo, N2L 3G1 Waterloo, Canada

toufik@math.haifa.edu,mschork@member.ams.org,simoseve@gmail.com

ABstract

Normally ordered forms of functions of boson operators are important in many contexts in particular concerning Quantum Field Theory and Quantum Optics. Beginning with the seminal work of Katriel [Lett. Nuovo Cimento 10(13):565-567, 1974], in the last few years, normally ordered forms have been shown to have a rich combinatorial structure, mainly in virtue of a link with the theory of partitions. In this paper, we attempt to enrich this link. By considering linear representations of noncrossing partitions, we define the notion of noncrossing normal ordering. Given the growing interest in noncrossing partitions, because of their many unexpected connections (like, for example, with free probability), noncrossing normal ordering appears to be an intriguing notion. We explicitly give the noncrossing normally ordered form of the functions $\left.\left(a^{r}\left(a^{\dagger}\right)^{s}\right)^{n}\right)$ and $\left(a^{r}+\left(a^{\dagger}\right)^{s}\right)^{n}$, plus various special cases. We are able to establish for the first time bijections between noncrossing contractions of these functions, $k$-ary trees and sets of lattice paths.

PACS NUMBERS: 02.10.Ox

\section{INTRODUCTION}

Let $a$ and $a^{\dagger}$ be boson annihilation and creation operators, satisfying the commutation relation $a a^{\dagger}-$ $a^{\dagger} a=1$. The normal ordering of an operator function $F\left(a, a^{\dagger}\right)$ consists in moving all creation operators to the left by applying the commutation relation. The resulting expression is called the normally ordered form of $F\left(a, a^{\dagger}\right)$ and it is denoted by $\mathcal{N}\left[F\left(a, a^{\dagger}\right)\right]$. The expressions $F\left(a, a^{\dagger}\right)$ and $\mathcal{N}\left[F\left(a, a^{\dagger}\right)\right]$, although generally different, represent the same function. The normally ordered form is particularly useful in quantum optics [23] and Quantum Field Theory [3]. On the basis of Wick's theorem [35], one can obtain $\mathcal{N}\left[F\left(a, a^{\dagger}\right)\right]$ from $F\left(a, a^{\dagger}\right)$ by means of two operations: contraction and double-dot operation. A contraction consists of substituting $a=1$ and $a^{\dagger}=1$ in an expression whenever $a$ precedes $a^{\dagger}$. An application of the double dot operation consists of deleting each occurrence of 1 and then arranging the expression so that $a^{\dagger}$ always precedes $a$. For example, $: a^{k}\left(a^{\dagger}\right)^{n}:=\left(a^{\dagger}\right)^{n} a^{k}$. Among all possible contractions, we also include the null contraction, that is the contraction leaving the expression invariant. Specifically,

$$
F\left(a, a^{\dagger}\right) \equiv \mathcal{N}\left[F\left(a, a^{\dagger}\right)\right]=\sum_{1}:\{\text { all possible contractions }\}: .
$$


For example, if $F\left(a, a^{\dagger}\right)=a a^{\dagger} a a a^{\dagger} a a$ then $\mathcal{N}\left[F\left(a, a^{\dagger}\right)\right]=\left(a^{\dagger}\right)^{2} a^{5}+4 a^{\dagger} a^{4}+2 a^{3}$.

The combinatorics of normally ordered forms has been studied in a number of papers (4 $]$ is a survey). For example, several authors established connections between Stirling, Bell numbers and normally ordered forms (see [20, 33] and references therein). In fact, it is nowadays well-known that $\mathcal{N}\left[\left(a^{\dagger} a\right)^{n}\right]=$ $\sum_{k=1}^{n} S(n, k)\left(a^{\dagger}\right)^{k} a^{k}$, where the integers $S(n, k)$ are the so called Stirling numbers of second kind (see, e.g., [30, Seq. A008277]), satisfying the recurrence relation $S(n+1, k)=k S(n, k)+S(n, k-1)$, with $S(n, 0)=\delta_{n, 0}$ and $S(n, k)=0$ for $k>n$. Generally, it can be difficult to obtain $\mathcal{N}\left[F\left(a, a^{\dagger}\right)\right]$ when $F\left(a, a^{\dagger}\right)$ is a polynomial of high order or an infinite series [11. A considerable amount of recent work has been produced in this direction [5, 6, 8, 9. Among the results, are explicit formulas for many examples of operators depending on $\left(a^{\dagger}\right)^{k} a^{n}$, characterized by integer powers $k$ and $n$, or depending on $q\left(a^{\dagger}\right) a+v\left(a^{\dagger}\right)$, with arbitrary functions $q$ and $v$.

The picture of this scenario has two faces: techniques from combinatorics are fruitfully applied to obtain normally ordered forms with immediate use in physics (e.g. the construction of generalized coherent states (7); the physical machinery behind this context helps to unveil and describe combinatorial properties (e.g. a theory of the Stirling and Bell polynomials can be formulated in terms of the algebraic and Fock space properties of the boson operators [20]).

The purpose of the present paper is to introduce and study the notion of noncrossing normally ordered form, an extremal structure with a well-defined combinatorial interpretation. Before entering this subject, it is useful to describe two ways to represent contractions: a graphical representation of contractions, which we call linear representation; a representation as words which is called canonical sequential form (see 24]). Let $\pi_{1} \pi_{2} \ldots \pi_{n}$ be a word whose letters are boson operators. Since our discussion is focused on the combinatorial properties of boson functions, we consider $a$ and $a^{\dagger}$ as letters, disregarding these as operators.

- Linear representation: We draw $n$ vertices, say $1,2, \ldots, n$, on a horizontal line, such that the point $i$ corresponds to the letter $\pi_{i}$; we represent each $a$ by a white vertex and each $a^{\dagger}$ by a black vertex; each black vertex $i$, incident with an edge, is connected by an undirected edge $(i, j)$ to a white vertex $j$. Importantly, the edges are drawn above the points. We call this graphical representation the linear representation of the contraction or, with an abuse of the terminology, just contraction. The linear representations of all contractions of the word $a a a^{\dagger} a^{\dagger}$ are illustrated in Figure 1.
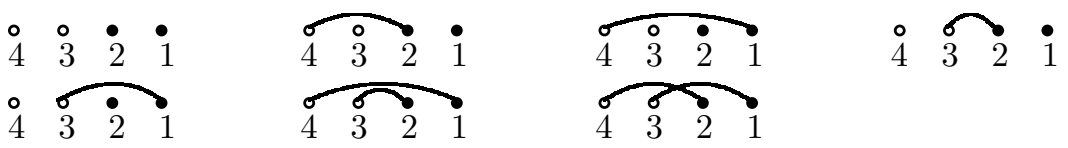

FIgURE 1. The linear representation of the contractions of the word $a a a^{\dagger} a^{\dagger}$

- Canonical sequential form: A contraction $\pi$ is represented by a sequence $a_{1} a_{2} \ldots a_{n}$ on the set $\left\{1,2, \ldots, n, 1^{\prime}, 2^{\prime}, \ldots, n^{\prime}\right\}$. In order to construct the sequence $a_{1} a_{2} \ldots a_{n}$, we need to read the contraction $\pi$ from right to left.

- If $\pi_{j}$ is a white (resp. black) vertex of degree 0 (that is, incident with no edges) we then replace it with $i^{\prime}$ (resp. $i$ ); $i$ is the smallest number not appearing in the sequence.

- If $\pi_{j}$ is a black vertex of degree 1 we then replace it with $i$, where $i$ is the smallest number not appearing in the sequence.

- If $\pi_{j}$ is a white vertex of degree 1 we then replace it with $i$, where $i$ is associated to the black vertex connected to $\pi_{j}$. 
For example, the contractions in Figure 1 can be represented by $123^{\prime} 4^{\prime}, 123^{\prime} 2,123^{\prime} 1,1223^{\prime}$, $1213^{\prime}, 1221$, and 1212. Such a representation is called the canonical sequential form [24].

We denote contractions also by enumerating the edges. For example, the contractions in Figure 1 are given by $\{\emptyset,(42),(41),(32),(31),(41)(32),(42)(31)\}$. Let $e=(i, j)$ and $e^{\prime}=(p, q)$ be two edges of a contraction $\pi$. We say that $e$ crosses $e^{\prime}$ if they intersect with each other, or, in other words, if $i<p<j<q$ or $p<i<q<j$. If this is the case, $e$ and $e^{\prime}$ are said to be a crossing of the contraction; otherwise, $e$ and $e^{\prime}$ are said to be a noncrossing. For example, from the contractions in Figure 1 only $(42)(31)$ is a crossing.

Using the linear representation form of contractions, the normally ordered form of an expression $F\left(a, a^{\dagger}\right)$ can be defined as follows. Given a contraction $\pi$ associated with its linear representation form, let freeb $(\pi)$ (resp. freew $(\pi)$ ) to be number black (resp. white) vertices of degree zero in $\pi$. The normally ordered form of $F\left(a, a^{\dagger}\right)$ is given by

$$
\sum_{\pi \text { is a contraction of } F\left(a, a^{\dagger}\right)}\left(a^{\dagger}\right)^{\text {freeb }(\pi)} a^{\text {freew }(\pi)} .
$$

A " $p$-analogue" of the normally ordered form can be defined as follows. Let us introduce an operator $P_{p}$ acting on each contraction $\pi$ of an expression $F=F\left(a, a^{\dagger}\right)$ :

$$
P_{p}(\pi):=p^{\operatorname{cross}(\pi)}\left(a^{\dagger}\right)^{\text {freeb }(\pi)} a^{\text {freew }(\pi)},
$$

where $\operatorname{cross}(\pi)$ counts the number of crossing edges in $\pi$. We extend $P_{p}$ to a linear operator by

$$
P_{p}(F):=\sum_{\pi} P_{p}(\pi)
$$

where the sum is taken over all contractions of the expression $F$. Note that the operator $P_{p}$ is a $p$-analogue of the standard double dot operation. Namely, for a given expression $F, P_{1}(F)$ is exactly the normally ordered form of $F$.

The number-states $|m\rangle$ are the states that satisfy $a^{\dagger} a|m\rangle=m|m\rangle$, where $\langle m \mid m\rangle=1$. The coherent states $|\gamma\rangle$ are the states that satisfy $a|\gamma\rangle=\gamma|\gamma\rangle$, where $\langle\gamma \mid \gamma\rangle=1$. Number-states and coherent states are important within the boson Fock space (see [3]). These are related by the wellknown expression $|\gamma\rangle=e^{-|\gamma|^{2} / 2} \sum_{m \geq 0} \frac{\gamma^{m}}{\sqrt{m !}}|m\rangle$. Many authors (see [5, 6, 7, 8, 9, 17, 18, 19, 20] ) dealt with explicit formulas for the expectation values $\left\langle\gamma\left|F\left(a, a^{\dagger}\right)\right| \gamma\right\rangle$. For example, Katriel [20] showed that $\left\langle\gamma\left|\left(a^{\dagger} a\right)^{n}\right| \gamma\right\rangle=\sum_{j=0}^{n} S(n, j)|\gamma|^{2 j}$, where $S(n, j)$ are the Stirling numbers of the second kind. This was done by proving that the normally ordered form of $\left(a^{\dagger} a\right)^{n}$ is given by $\sum_{j=0}^{n} S(n, j)\left(a^{\dagger}\right)^{j} a^{j}$. By applying our operator $P_{p}$ to the expression $\left(a^{\dagger} a\right)^{n}$, one can write

$$
P_{p}\left(\left(a^{\dagger} a\right)^{n}\right)=\sum_{j=0}^{n} f_{n, j}(p)\left(a^{\dagger}\right)^{j} a^{j}
$$

where $f_{n, j}(p)$ is a polynomial in $p$ (note that $f_{n, j}(1)=S(n, j)$ ). In this paper, we study the combinatorial structure of $P_{0}(F)$. In particular, with Theorem 3, we show that

$$
P_{0}\left(\left(a a^{\dagger}\right)^{n}\right)=\sum_{j=0}^{n} \frac{1}{n+1}\left(\begin{array}{c}
n+1 \\
j+1
\end{array}\right)\left(\begin{array}{c}
n+1 \\
j
\end{array}\right)\left(a^{\dagger}\right)^{n-j} a^{n-j}
$$

If the commutation relations hold for the operators then one has $a a^{\dagger}=a^{\dagger} a+1$ and, therefore, a close relation between $\left(a a^{\dagger}\right)^{n}$ and $\left(a^{\dagger} a\right)^{n}$. 
TOUFIK MANSOUR, MATTHIAS SCHORK AND SIMONE SEVERINI

A noncrossing contraction is a contraction whose edges are all noncrossing. Figure 1 gives all contractions of the word $a a a^{\dagger} a^{\dagger}$ : these consist of 1 crossing and 6 noncrossing contractions (one of those is the null contraction). With this terminology, we are now ready to give the definition of noncrossing normal ordering.

Definition. Let $F\left(a, a^{\dagger}\right)=\sum_{w \in A} w$ be any expression composed by elements of $A$, where $A$ is any finite set of finite words on the alphabet $\left\{a, a^{\dagger}\right\}$. The noncrossing normally ordered form of $F\left(a, a^{\dagger}\right)$ is denoted and defined by

$$
\mathcal{N C}\left(F\left(a, a^{\dagger}\right)\right)=\sum:\{\text { all possible noncrossing contractions }\}:
$$

It is important to note that $P_{0}\left(F\left(a, a^{\dagger}\right)\right)=\mathcal{N C}\left(F\left(a, a^{\dagger}\right)\right)$. The central concept of the paper is then a "noncrossing combinatorial structure". A number of aspects of noncrossing combinatorial structures have been studied in recent years. In particular, noncrossing partitions have recently turned out to be a flourishing subject, given the links with other concepts like free probability, parking functions, and braid groups (see [26, 29]). Specifically, in enumerative combinatorics, Klazar linked noncrossing partitions to the well-known Catalan numbers (or the lattice Dyck paths) 24, 25], while other authors have related noncrossing partitions to RNA secondary structure and Motzkin paths (e.g., see [12] and references therein). Here, we apply the notion of linear representation of noncrossing partitions to contractions of normally ordered forms. In this way, the obtained noncrossing normally ordered forms can be related to a number of different combinatorial objects. Namely, we study $\mathcal{N C}\left(\left(a^{r}\left(a^{\dagger}\right)^{s}\right)^{n}\right)$ (in Section 3), $\mathcal{N C}\left(\left(a^{r}+\left(a^{\dagger}\right)^{s}\right)^{n}\right)$ (in Section 4) and some special cases. In Section 5, we establish bijections between sets of noncrossing contractions of special cases of these functions (for example, $\left(a+\left(a^{\dagger}\right)^{2}\right)^{n},\left(a^{r} a^{\dagger}\right)^{n}$ and $\left.\left(a\left(a^{\dagger}\right)^{r}\right)^{n}\right)$ sets of trees and sets of lattice paths (for example, $k$-ary trees and 2-Motzkin paths). Section 6, the last section of the paper, is devoted to draw some directions for further analysis. Physical interpretations of the noncrossing normal ordering remain as desiderata.

\section{SOME PRELIMINARY OBSERVATIONS}

Let us denote for arbitrary $p$ the corresponding normal ordering by

$$
\mathcal{N}_{p}\left(F\left(a, a^{\dagger}\right)\right) \equiv P_{p}\left(F\left(a, a^{\dagger}\right)\right)
$$

such that $\mathcal{N}_{1} \equiv \mathcal{N}$ (the conventional normal ordering) and $\mathcal{N}_{0} \equiv \mathcal{N C}$ (the noncrossing normal ordering). If we denote the associated coefficients by $C_{F ; k, l}(p)$, i.e.,

$$
\mathcal{N}_{p}\left(F\left(a, a^{\dagger}\right)\right)=\sum_{k, l} C_{F ; k, l}(p)\left(a^{\dagger}\right)^{k} a^{l},
$$

then it is clear that one has for any $F$ and all $k, l$ the following inequality

$$
0 \leq C_{F ; k, l}(0) \leq C_{F ; k, l}(1) .
$$

For example, if $F\left(a, a^{\dagger}\right)=\left(a^{\dagger} a\right)^{n}$ then one has $C_{\left(a^{\dagger} a\right)^{n} ; k, l}(1)=S(n, k) \delta_{k l}$.

Example 1. Let $F\left(a, a^{\dagger}\right)=a a a^{\dagger} a^{\dagger}$ be the example of Figure 1. Using the commutation relations or (11) one may show that $\mathcal{N}_{1}\left(a a a^{\dagger} a^{\dagger}\right)=\left(a^{\dagger}\right)^{2} a^{2}+4 a^{\dagger} a+2$. Since there is exactly one crossing contraction of degree two, namely $(42)(31)$, one has $\mathcal{N}_{0}\left(a a a^{\dagger} a^{\dagger}\right)=\left(a^{\dagger}\right)^{2} a^{2}+4 a^{\dagger} a+1$. 
Generalizing the observation of the preceding example, it is clear that $\mathcal{N}_{0}\left(F\left(a, a^{\dagger}\right)\right) \neq \mathcal{N}_{1}\left(F\left(a, a^{\dagger}\right)\right)$ if and only if $F\left(a, a^{\dagger}\right)$ is a polynomial of degree at least two in $a$ and $a^{\dagger}$. In particular, for lower degrees the two expressions coincide. This has the consequence that $\mathcal{N}_{0}\left(a a^{\dagger}\right)=a^{\dagger} a+1$. Recall that in the conventional case one also has $\mathcal{N}_{1}\left(a a^{\dagger}\right)=a^{\dagger} a+1$ directly from (11). On the other hand, one can use the commutation relation to find the same result, i.e., $\mathcal{N}_{1}\left(a a^{\dagger}\right)=\mathcal{N}_{1}\left(a^{\dagger} a+1\right)=a^{\dagger} a+1$. Let us try to reproduce the noncrossing statistics according to (2) by modifying the commutation relations and using the usual normal ordering process. Thus, we consider operators $b, b^{\dagger}$ satisfying

$$
b b^{\dagger}-\kappa b^{\dagger} b=\lambda_{1} b^{\dagger}+\lambda_{2} b+\lambda_{3}
$$

where $\kappa$ and $\lambda_{i}$ are some parameters (here we have assumed that the right-hand side has lower degree than the left-hand side). Using this commutator, it follows that

$$
\mathcal{N}_{0}\left(b b^{\dagger}\right) \stackrel{3}{=} \mathcal{N}_{0}\left(\kappa b^{\dagger} b+\lambda_{1} b^{\dagger}+\lambda_{2} b+\lambda_{3}\right)=\kappa b^{\dagger} b+\lambda_{1} b^{\dagger}+\lambda_{2} b+\lambda_{3}
$$

where we have used in the second equation the fact that $\mathcal{N}_{0}$ is linear and that all summands are already normally ordered. However, if this has to be the result obtained from the definition (2) then one has neccessarily $\kappa=1=\lambda_{3}$ as well as $\lambda_{1}=\lambda_{2}=0$, reproducing for $b, b^{\dagger}$ the conventional commutation relations. However, the explicit examples show that the operators $b, b^{\dagger}$ cannot satisfy the conventional commutation relations and the noncrossing normal ordering (2) simultanously! Thus, it is not clear whether one should speak of the operators $a, a^{\dagger}$ - whose words $F\left(a, a^{\dagger}\right)$ are brought into noncrossing normally ordered form using (2) - as "bosonic" operators anymore. It would be very interesting to find the algebraic relations which the operators have to satisfy such that normal ordering using these algebraic relations is equivalent to the definition (2). A discussion of a general approach to generalizations of normal orderings using nearly arbitrary weights can be found in 27 .

Let us mention that very closely related situations have been considered in the context of $q$-Fock spaces and $q$-Gaussian processes when the limit $q \rightarrow 0$ is considered, see, e.g., [1, 10, 15] and the references given therein. Here the corresponding annihilation and creation operators satisfy the $q$ deformed commutation relations. In the limit $q \rightarrow 0$ one considers full Fock space and there exists an intimate link between normal ordered representations and noncrossing partitions.

\section{Noncrossing NORMAL ORDERING OF $\left(a^{r}\left(a^{\dagger}\right)^{s}\right)^{n}$}

Let us denote by $\mathcal{V}_{r s}(n)$ the set of all the linear representations of the noncrossing contractions of $\left(a^{r}\left(a^{\dagger}\right)^{s}\right)^{n}$. For each linear representation $\pi \in \mathcal{V}_{r s}(n)$, define $e(\pi)$ to be the number of edges in $\pi$. Let $B_{r, s}(x, y)$ be the generating function for the number of linear representations $\pi \in \mathcal{V}_{r s}(n)$ with exactly $m$ edges, that is,

$$
B_{r, s}(x, y)=\sum_{n \geq 0} \sum_{\pi \in \mathcal{V}_{r s}(n)} x^{n} y^{e(\pi)} .
$$

The noncrossing normally ordered form of $\left(a^{r}\left(a^{\dagger}\right)^{s}\right)^{n}$ is given by

$$
\mathcal{N C}\left(\left(a^{r}\left(a^{\dagger}\right)^{s}\right)^{n}\right)=\sum_{j \geq 0}\left(\text { the coefficient of } x^{n} y^{j} \text { in } B_{r, s}(x, y)\right)\left(a^{\dagger}\right)^{s n-j} a^{r n-j} .
$$

So, to find the noncrossing normally ordered form of $\left(a^{r}\left(a^{\dagger}\right)^{s}\right)^{n}$, it is enough to find an explicit formula for the generating function $B_{r, s}(x, y)$. In this section, we present a nonlinear system of equations whose solution gives an explicit formula for $B_{r, s}(x, y)$. We begin by writing

$$
B_{r, s}(x, y)=1+B_{r, s}(x, y ; s),
$$


where $B_{r, s}(x, y ; t)$ is the generating function for all the linear representations $\pi=\pi_{1} \ldots \pi_{n} \in \mathcal{V}_{r s}(n)$, such that the canonical sequential form of $\pi$ starts with $12 \ldots t$. With the following lemma, we give a recurrence relation for the sequence $B_{r, s}(x, y ; t)$.

Lemma 2. Let $z^{\prime}=x^{\frac{1}{r+s}}$. For all $t=1,2, \ldots, s$,

$$
B_{r, s}(x, y ; t)=z^{\prime} B_{r, s}(x, y ; t-1)+B_{r, s}(x, y) \sum_{j=1}^{r} z^{\prime r-j} B_{r, s}(x, y ; j, t),
$$

with the initial condition $B_{r, s}(x, y ; 0)=z^{\prime r} B_{r, s}(x, y)$, where $B_{r, s}(x, y ; a, b)$ is the generating function for the number of linear representations $\pi \in \mathcal{V}_{r s}(n)$ such that $\pi$ starts with $b$ black vertices and end with a white vertices and there is an edge between the first black vertex and last white vertex.

Proof. Let $\pi$ be any linear representations in $\mathcal{V}_{r s}(n)$ such that $\pi$ starts with $t$ black vertices. Let us write an equation for $B_{r, s}(x, y ; t)$. The first black vertex has degree either zero or one. The contribution of the first case is

$$
z^{\prime} B_{r, s}(x, y ; t-1)
$$

Now, let us consider the second case, that is the first black vertex having degree one. The contraction $\pi$ can be written as $\pi=\pi^{\prime}(p+1)^{\prime} \ldots q^{\prime} \pi^{\prime \prime}$, where $\pi^{\prime}$ starts with $t$ black vertices, ends with $j$ white vertices and there is an edge between the first black vertex of $\pi^{\prime}$ and the last white vertex of $\pi^{\prime}$. Moreover, $\pi^{\prime}$ is followed by $q-p$ white vertices such that $q-p+j=r$, see the following figure:

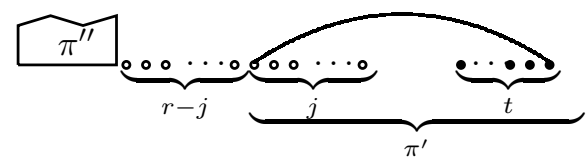

Since we are interested in the noncrossing contractions, we may observe there are no edges from $\pi^{\prime}(p+1)^{\prime} \ldots q^{\prime}$ to $\pi^{\prime \prime}$. Therefore, the contribution of the second case gives

$$
B_{r, s}(x, y) \sum_{j=1}^{r} z^{\prime r-j} B_{r, s}(x, y ; j, t) .
$$

Considering these disjoint cases together we arrive to our recurrence relation. Since there are no edges from white vertices to other vertices, the generating function is $B_{r, s}(x, y ; 0)=z^{\prime r} B_{r, s}(x, y)$, as required by the statement of the lemma.

As we have seen in Lemma 2, to find a formula for the generating function $B_{r, s}(x, y)$, we need to have a recurrence relation for the generating functions $B_{r, s}(x, y ; p, q)$. This is done as follows.

Lemma 3. Let $z^{\prime}=x^{\frac{1}{r+s}}, p=2,3, \ldots, r$, and $q=2,3, \ldots, s$. Then the following hold:

(i) $B_{r, s}(x, y ; 1,1)=y z^{\prime 2}+x y z^{2} B_{r, s}(x, y)$.

(ii) $B_{r, s}(x, y ; p, 1)=z^{\prime} B_{r, s}(x, y ; p-1,1)+y z^{\prime r+2} B_{r, s}(x, y) \sum_{j=1}^{s}{z^{\prime}}^{s-j} B_{r, s}(x, y ; p-1, j)$.

(iii) $B_{r, s}(x, y ; 1, q)=z^{\prime} B_{r, s}(x, y ; 1, q-1)+y{z^{\prime}}^{s+2} B_{r, s}(x, y) \sum_{j=1}^{r} z^{\prime r-j} B_{r, s}(x, y ; j, q-1)$.

$$
\begin{aligned}
B_{r, s}(x, y ; p, q)=z^{\prime} B_{r, s}(x, y ; p-1, q) & +y z^{\prime 2} \sum_{j=1}^{q-1} z^{\prime q-1-j} B_{r, s}(x, y ; p-1, j) \\
& +y z^{\prime 2} B_{r, s}(x, y ; q-1) \sum_{j=1}^{s} z^{\prime s-j} B_{r, s}(x, y ; p-1, j) .
\end{aligned}
$$


Proof. Let $\pi$ be any linear representations in $\mathcal{V}_{r s}(n)$ such that the last $p$ vertices of $\pi$ are white, the first $q$ vertices of $\pi$ are black and there is an edge between the first black vertex and the last white vertex. The generating function for the number of such linear representations $\pi$ is given by $B_{r, s}(x, y ; p, q)$. Now, let us write an equation for $B_{r, s}(x, y ; p, q)$ for each of the following four cases:

- If $p=q=1$ then there are two possibilities for the linear representation $\pi: \pi=11$ or $\pi=12^{\prime} 3^{\prime} \ldots r^{\prime} \pi^{\prime}(d+1)(d+2) \ldots(d+s) 1$. The first contribution gives $z^{\prime 2} y$ and the second contribution gives $y z^{\prime r+s+2} B_{r, s}(x, y)=y x z^{\prime 2} B_{r, s}(x, y)$. Putting together the two disjoint cases we obtain (i).

- If $p \geq 2$ and $q=1$ then the degree of the vertex $v$, the one before the last white vertex (which is also a white vertex), is either zero or one. The first contribution gives $z^{\prime} B_{r, s}(x, y ; p-1,1)$. In the second case, there exists a black vertex connected to $v$. Then $\pi$ can be represented as

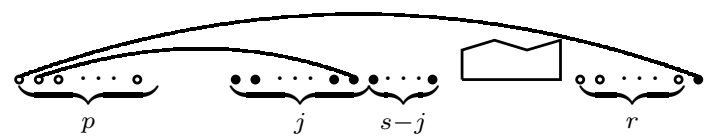

Thus, this contribution gives $y z^{\prime r+2} B_{r, s}(x, y) \sum_{j=1}^{s} z^{\prime s-j} B_{r, s}(x, y ; p-1, j)$. Putting together the two disjoint cases above, we obtain (ii), as required.

- If $q \geq 2$ and $p=1$ then the degree of $v$, the second vertex (which is black), is either zero or one. The first contribution gives $z^{\prime} B_{r, s}(x, y ; 1, q-1)$. In the second case, there exists a white vertex connected to $v$. Then $\pi$ can be represented as

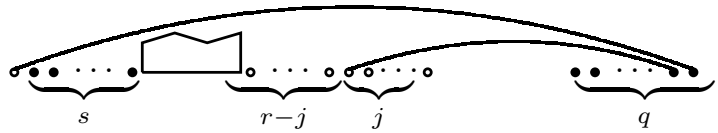

Thus, this contribution gives $y z^{\prime s+2} B_{r, s}(x, y) \sum_{j=1}^{r} z^{\prime r-j} B_{r, s}(x, y ; j, q-1)$. Putting together these two disjoint cases, we get $(i i)$, as requested.

- Let $p, q \geq 2$. We consider the following two cases corresponding to the possible degrees of $v$, the vertex (which is white) before the last vertex. The contribution of the case in which the degree of $v$ is zero gives $z^{\prime} B_{r, s}(x, y ; p-1, q)$. If the degree of $v$ is one, then there exists a black vertex $w$ connected to $v$. Then there are two possibilities: $w$ is one of the first $q-1$ black vertices or is not one of those. The contribution of the first case gives $y z^{\prime 2} \sum_{j=1}^{q-1} z^{q-1-j} B_{r, s}(x, y ; p-1, j)$. The contribution of the second case (similar to the case $p \geq 2$ and $q=1)$ gives $y z^{\prime 2} B_{r, s}(x, y ; q-1) \sum_{j=1}^{s} z^{\prime s-j} B_{r, s}(x, y ; p-1, j)$. Putting together the two disjoint cases above, we obtain (iv), as claimed.

Lemma 2 and Lemma 3 together with Eq. (4) give a (nonlinear) system of equations in the variables $B_{r, s}(x, y), B_{r, s}(x, y ; t)(t=0,1, \ldots, s)$, and $B_{r, s}(x, y ; p, q)(p=1,2, \ldots, r$ and $q=1,2, \ldots, s)$. We solve this system for several interesting cases.

Theorem 4. Let $r \geq 1$. Then

$$
B_{r, 1}(x, y)=B_{1, r}(x, y)=\left(1+x B_{1, r}(x, y)\right)\left(1+x y B_{1, r}(x, y)\right)^{r} .
$$


Moreover, for all $n \geq 0$,

$$
\begin{aligned}
& \mathcal{N C}\left(\left(a^{r} a^{\dagger}\right)^{n}\right)=\sum_{j=0}^{n} \frac{1}{n+1}\left(\begin{array}{c}
n+1 \\
j+1
\end{array}\right)\left(\begin{array}{c}
r n+r \\
j
\end{array}\right)\left(a^{\dagger}\right)^{n-j} a^{r n-j} \\
& \mathcal{N C}\left(\left(a\left(a^{\dagger}\right)^{r}\right)^{n}\right)=\sum_{j=0}^{n} \frac{1}{n+1}\left(\begin{array}{c}
n+1 \\
j+1
\end{array}\right)\left(\begin{array}{c}
r n+r \\
j
\end{array}\right)\left(a^{\dagger}\right)^{r n-j} a^{n-j}
\end{aligned}
$$

The above theorem can be proved combinatorially as described in Section 5. Another application of Lemma 2 and Lemma 3 is the next observation.

Theorem 5. The generating function $B_{2,2}(x, y)$ satisfies

$$
\begin{aligned}
B_{2,2}(x, y)=1+x(1+y)^{2} B_{2,2}(x, y) & +2 x y\left(1+x(1+y)+x y^{2}\right) B_{2,2}^{2}(x, y) \\
& +x^{2} y^{2}\left(x(1+y)^{2}-1\right) B_{2,2}^{3}(x, y)+x^{4} y^{4} B_{2,2}^{4}(x, y) .
\end{aligned}
$$

\section{NONCROSSING NORMAL ORDERING FORM OF $\left(a^{r}+\left(a^{\dagger}\right)^{s}\right)^{n}$}

Let us denote by $\mathcal{W}_{r s}(n)$ the set of all the linear representations of the noncrossing contractions of $\left(a^{r}+\left(a^{\dagger}\right)^{s}\right)^{n}$. For each linear representation $\pi \in \mathcal{W}_{r s}(n)$, define $w(\pi)$ (resp. $e(\pi)$ ) to be the number of white vertices (resp. edges) in $\pi$ and. Let $A_{r, s}(x, y, z)$ be the generating function for the number of linear representations $\pi \in \mathcal{W}_{r s}(n)$ with exactly $m$ edges and $d$ white vertices, that is,

$$
A_{r, s}(x, y, z)=\sum_{n \geq 0} \sum_{\pi \in \mathcal{W}_{r s}(n)} x^{n} y^{e(\pi)} z^{w(\pi)} .
$$

Hence, the noncrossing normally ordered form of $\left(a^{r}+\left(a^{\dagger}\right)^{s}\right)^{n}$ is given by

$$
\mathcal{N C}\left(\left(a^{r}+\left(a^{\dagger}\right)^{s}\right)^{n}\right)=\sum_{i \geq 0} \sum_{j=0}^{i}\left(\text { the coefficient of } x^{n} y^{j} z^{i} \text { in } A_{r, s}(x, y, z)\right)\left(a^{\dagger}\right)^{n-r i-j} a^{i-j}
$$

In order to find the noncrossing normally ordered form of $\left(a^{r}+\left(a^{\dagger}\right)^{s}\right)^{n}$, it is enough to find an explicit formula for the generating function $A_{r, s}(x, y, z)$. In this section, we present a nonlinear system of equations whose solution gives an explicit formula for $A_{r, s}(x, y, z)$. We write

$$
A_{r, s}(x, y, z)=1+x z^{r} A_{r, s}(x, y, z)+A_{r, s}(x, y, z ; s),
$$

where $A_{r, s}(x, y, z ; t)$ is the generating function for all the linear representations $\pi \in \mathcal{W}_{r s}(n)$ such that the canonical sequential form of $\pi$ starts with $12 \ldots t$. Applying a similar argument as in the proof of Lemma 2, we have a recurrence relation for the sequence $A_{r, s}(x, y, z ; t)$.

Lemma 6. Let $z^{\prime}=x^{\frac{1}{s}}$ and $z^{\prime \prime}=x^{\frac{1}{r}}$. For all $t=1,2, \ldots, s$,

$$
A_{r, s}(x, y, z ; t)=z^{\prime} A_{r, s}(x, y, z ; t-1)+A_{r, s}(x, y, z) \sum_{j=1}^{r}\left(z^{\prime \prime} z\right)^{r-j} A_{r, s}(x, y, z ; j, t)
$$

with the initial condition $A_{r, s}(x, y, z ; 0)=A_{r, s}(x, y, z)$, where $A_{r, s}(x, y ; a, b)$ is the generating function for the number of linear representations $\pi \in \mathcal{W}_{r s}(n)$, such that $\pi$ starts with $b$ black vertices, ends with a white vertices and there is an edge between the first black vertex and last white vertex.

Proof. Let $\pi$ be any linear representation in $\mathcal{W}_{r s}(n)$ such that $\pi$ starts with $t$ black vertices. Let us write an equation for $B_{r, s}(x, y ; t)$. The first black vertex has degree either zero or one. The contribution of the first case is $z^{\prime} A_{r, s}(x, y, z ; t-1)$. Now let us consider the second case, that is, the first black vertex has degree one. 


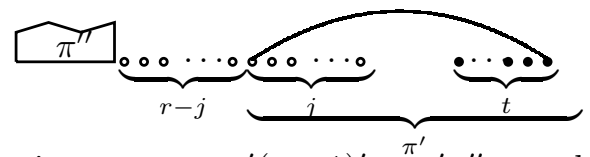

Thus our contraction $\pi$ can be written as $\pi=\pi^{\prime}(p+1)^{\prime} \ldots{ }^{\pi^{\prime}} q^{\prime} \pi^{\prime \prime}$, see the above figure, where $\pi^{\prime}$ starts with $t$ black vertices and ends with $j$ white vertices; there is an edge between the first back vertex of $\pi^{\prime}$ and the last white vertex of $\pi^{\prime} ; \pi^{\prime}$ is followed by $q-p$ white vertices such that $q-p+j=r$. Since we are interested in the noncrossing contractions, there are no edges from $\pi^{\prime}(p+1)^{\prime} \ldots q^{\prime}$ and $\pi^{\prime \prime}$. Therefore, the contribution of this second case gives

$$
A_{r, s}(x, y, z) \sum_{j=1}^{r}\left(z z^{\prime \prime}\right)^{r-j} A_{r, s}(x, y, z ; j, t) .
$$

Adding the above disjoint cases we obtain the recurrence relation. From the definitions, we obtain that $A_{r, s}(x, y, z ; 0)=A_{r, s}(x, y, z)$, as required.

As we see in Lemma 6, to find a formula for the generating function $A_{r, s}(x, y, z)$, we need to find a recurrence relation for the generating functions $A_{r, s}(x, y, z ; p, q)$. This can be done by using a similar techniques as in the proof of Lemma 3. which gives a recurrence relation for the sequence $A_{r, s}(x, y, z ; p, q)$.

Lemma 7. Let $z^{\prime}=x^{\frac{1}{s}}$ and $z^{\prime \prime}=x^{\frac{1}{r}}, p=2,3, \ldots, r$, and $q=2,3, \ldots, s$. Then the following holds:

(i) $A_{r, s}(x, y, z ; 1, q)=y z z^{\prime} z^{\prime \prime} A_{r, s}(x, y, z ; q-1)$.

$$
\begin{array}{r}
A_{r, s}(x, y, z ; p, q)=y z z^{\prime} z^{\prime \prime} A_{r, s}(x, y, z ; p-1, q)+y z^{\prime} z^{\prime \prime} \sum_{j=1}^{q-1} z^{\prime q-1-j} A_{r, s}(x, y, z ; p-1, j) \\
+y z z^{\prime} z^{\prime \prime} A_{r, s}(x, y, z ; q-1) \sum_{j=1}^{s} z^{\prime s-j} A_{r, s}(x, y, z ; p-1, j) .
\end{array}
$$

Proof. Let $\pi$ be any linear representations in $\mathcal{W}_{r s}(n)$ such that the last $p$ vertices of $\pi$ are white, the first $q$ vertices of $\pi$ are black, and there is an edge between the first black vertex and the last white vertex. The generating function for the number of such linear representations $\pi$, respect to the number vertices, the number of white vertices and the number edges in $\pi$, is given by $A_{r, s}(x, y, z ; p, q)$. Now, let us write an equation for $A_{r, s}(x, y, z ; p, q)$ for each of the following two cases:

- If $p=1$ and $q \geq 1$ then it is not hard to see from the definitions that $A_{r, s}(x, y, z ; 1, q)=$ $y z z^{\prime} z^{\prime \prime} A_{r, s}(x, y, z ; q-1)$.

- Let $p \geq 2$. Let us consider the following two cases that correspond to the degree of $v$, the vertex before the last vertex (which is white). The contribution of the case when the degree of $v$ is zero gives $y z z^{\prime} z^{\prime \prime} A_{r, s}(x, y, z ; p-1, q)$. If degree of $v$ is one then there exists a black vertex $w$ connected by an edge with $v$. There are two possibilities: $w$ is one of the first $q-1$ black vertices or it is not. Applying similar arguments as in the proof of Lemma 3, we can see that the contribution of the first case gives

$$
y z^{\prime} z^{\prime \prime} \sum_{j=1}^{q-1} z^{q-1-j} A_{r, s}(x, y, z ; p-1, j)
$$

and the contribution of the second case gives

$$
y z z^{\prime} z^{\prime \prime} A_{r, s}(x, y, z ; q-1) \sum_{j=1}^{s} z^{\prime s-j} A_{r, s}(x, y, z ; p-1, j) .
$$


Together, the two case above give (ii), as claimed.

Hence, Lemma 6] and Lemma 7 together with (5) gives a (nonlinear) system of equations in the variables $A_{r, s}(x, y, z), A_{r, s}(x, y, z ; t)(t=0,1, \ldots, s)$, and $A_{r, s}(x, y, z ; p, q)(p=1,2, \ldots, r$ and $q=1,2, \ldots, s)$.

Theorem 8. The generating function $A=A_{r, 1}(x, y, z)$ satisfies

$$
A=1+x\left(1+z^{r}\right) A+x^{2} y z^{r} A^{r} \frac{\left.1-x^{r} y^{r}(1+A)^{r}\right)}{1-x y(1+A)} .
$$

For example, when $r=1$, the above theorem gives

$$
A_{1,1}(x, y, z)=\frac{1-x z-x-\sqrt{(1-x-x z)^{2}-4 x^{2} y z}}{2 x^{2} y z} .
$$

Using the fact that $\frac{1-\sqrt{1-4 x}}{2 x}=\sum_{n \geq 0} c_{n} x^{n}$, where $c_{n}=\frac{1}{n+1}\left(\begin{array}{c}2 n \\ n\end{array}\right)$ the $n$-th Catalan number, we have

$$
A_{1,1}(x, y, z)=\sum_{n \geq 0} \sum_{j=0}^{n} \sum_{i=j}^{n-j} c_{j}\left(\begin{array}{c}
n \\
i+j
\end{array}\right)\left(\begin{array}{c}
i+j \\
2 j
\end{array}\right) x^{n} y^{j} z^{i} .
$$

Thus, the noncrossing normally ordered form of $\left(a+a^{\dagger}\right)^{n}$ is given by

$$
\mathcal{N C}\left(\left(a+a^{\dagger}\right)^{n}\right)=\sum_{j=0}^{n} \sum_{i=j}^{n-j} c_{j}\left(\begin{array}{c}
n \\
i+j
\end{array}\right)\left(\begin{array}{c}
i+j \\
2 j
\end{array}\right)\left(a^{\dagger}\right)^{n-i-j} a^{i-j}
$$

Another application of Lemma 6 and Lemma 7 with $r=1$ and $s \geq 1$ is that the generating function $A_{1, s}(x, y, z)$ satisfies the equation

$$
A_{1, s}(x, y, z)=1+x z A_{1, s}(x, y, z)+x A_{1, s}(x, y, z)\left(1+x y z A_{1, s}(x, y, z)\right)^{s} .
$$

By Lagrange inversion formula 36] on the above equation, we obtain that

$$
A_{1, s}(x, y, z)=\sum_{n \geq 0} \sum_{j=0}^{n} \sum_{i=j}^{n} \frac{1}{n+1}\left(\begin{array}{c}
n+1 \\
j+1
\end{array}\right)\left(\begin{array}{c}
n-j \\
n-i
\end{array}\right)\left(\begin{array}{c}
s(n-i) \\
j
\end{array}\right) x^{n} y^{j} z^{i},
$$

which leads to the following result.

Theorem 9. The normally ordered form of $\left(a+\left(a^{\dagger}\right)^{s}\right)^{n}$ is given by

$$
\mathcal{N C}\left(\left(a+\left(a^{\dagger}\right)^{s}\right)^{n}\right)=\sum_{j=0}^{n} \sum_{i=j}^{n} \frac{1}{n+1}\left(\begin{array}{c}
n+1 \\
j+1
\end{array}\right)\left(\begin{array}{c}
n-j \\
n-i
\end{array}\right)\left(\begin{array}{c}
s(n-i) \\
j
\end{array}\right)\left(a^{\dagger}\right)^{s(n-i)-j} a^{i-j} .
$$

We remark that Theorem 9 for $s=1$ gives

$$
\begin{aligned}
\mathcal{N C} & \left(\left(a+a^{\dagger}\right)^{n}\right) \\
& =\sum_{j=0}^{n} \sum_{i=j}^{n-j} \frac{1}{n+1}\left(\begin{array}{c}
n+1 \\
j+1
\end{array}\right)\left(\begin{array}{c}
n-j \\
n-i
\end{array}\right)\left(\begin{array}{c}
n-i \\
j
\end{array}\right)\left(a^{\dagger}\right)^{n-i-j} a^{i-j}=\sum_{j=0}^{n} \sum_{i=j}^{n-j} \frac{n !}{j !(j+1) !(i-j) !(n-i-j) !}\left(a^{\dagger}\right)^{n-i-j} a^{i-j} \\
& =\sum_{j=0}^{n} \sum_{i=j}^{n-j} \frac{(2 j) !}{j !(j+1) !} \frac{n !}{(i+j) !(n-i-j) !} \frac{(i+j) !}{(2 j) !(i-j) !}\left(a^{\dagger}\right)^{n-i-j} a^{i-j}=\sum_{j=0}^{n} \sum_{i=j}^{n-j} c_{j}\left(\begin{array}{c}
n \\
i+j
\end{array}\right)\left(\begin{array}{c}
i+j \\
2 j
\end{array}\right)\left(a^{\dagger}\right)^{n-i-j} a^{i-j},
\end{aligned}
$$

as described in (6). 


\section{Two Particular CASEs, $k$-ARy trees, AND lattice Paths}

In this section, we relate our linear representations $\mathcal{V}_{r s}(n)$ and $\mathcal{W}_{r s}(n)$, for several particular cases of $r$ and $s$, to different combinatorial structure, such as $k$-ary trees, lattice paths, and Dyck paths (these notions will be defined below).

5.1. The case $\left(a^{r} a^{\dagger}\right)^{n}$ and $k$-ary trees. Our goal is to show that the number of noncrossing contractions of $\left(a^{r} a^{\dagger}\right)^{n}$ is counted by the generalized Catalan numbers $C_{n, k}$, defined by $C_{n, k}=\frac{1}{(k-1) n+1}\left(\begin{array}{c}k n \\ n\end{array}\right)$. In our approach, we give a recursive construction of the set $\mathcal{V}_{r 1}(n)$. Intuitively, for a noncrossing contraction $\pi=\pi_{1} \pi_{2} \cdots \pi_{n}$ in $\mathcal{V}_{r 1}(n)$, we may obtain a contraction in $\mathcal{V}_{r 1}(n-1)$. Then we need to keep track of all possible ways to recover a noncrossing contraction in $\mathcal{V}_{r 1}(n)$ from a smaller noncrossing contraction. In the recursive generation of noncrossing contractions with $n$ vertices, one is often concerned with the edges whose points have the label 1 in the canonical sequential form. However, for the purpose of this paper, we consider edges whose first point is labeled by 1 . We denote by $E_{\pi}$ the edge $(1, j)$. In general, we use the notation $E_{i}$ to denote the edge with first point $i$. In this sense, $E_{\pi}=E_{1}$. Also, we make use of the ordered set $F_{\pi}$ of all white vertices $j-1, j-2, \ldots, i+1$, where $i$ is maximal, $i<j$ and $i$ is a black vertex.

An edge $(i, j)$ is said to cover the edge $\left(i^{\prime}, j^{\prime}\right)$ if and only if $i<i^{\prime}<j^{\prime}<j$. We have the following lemma on the structure of the noncrossing contractions of $\left(a^{r} a^{\dagger}\right)^{n}$. The lemma is straightforward to prove.

Lemma 10. Let $\pi$ be any noncrossing contraction in the set $\mathcal{V}_{r 1}(n)$. Then the canonical subsequential form of $\pi$ is

$$
\begin{aligned}
& a_{1}\left(a_{1}+1\right)^{\prime} \ldots\left(a_{1}+r\right)^{\prime} \pi^{(1)} \ldots a_{m-1}\left(a_{m-1}+1\right)^{\prime} \ldots\left(a_{m-1}+r\right)^{\prime} \pi^{(m-1)} \\
& a_{m} \pi^{(m)}\left(a_{m}+s_{0}\right)^{\prime} \ldots\left(a_{m}+s_{1}-1\right)^{\prime} a_{m}\left(a_{m}+s_{1}\right)^{\prime} \ldots\left(a_{m}+s_{2}-1\right)^{\prime} \\
& \quad a_{m-1} \cdots\left(a_{m}+s_{m-1}\right)^{\prime} \ldots\left(a_{m}+s_{m}-1\right)^{\prime} a_{1}\left(a_{m}+s_{m}\right)^{\prime} \ldots\left(a_{m}+s_{m+1}-1\right)^{\prime} \pi^{(m+1)}
\end{aligned}
$$

where $a_{1}=1, a_{i+1}-a_{i}>r, s_{i+1} \geq s_{i} \geq 0, \pi^{(i)} \in \mathcal{V}_{r 1}\left(a_{i+1}-a_{i}-r-1\right), i=1,2, \ldots, m-1, \pi^{(m)}$ is either empty $\left(s_{0}=1\right)$ or $\pi^{(m)}=\left(a_{m}+1\right)^{\prime} \ldots\left(a_{m}+r\right)^{\prime} \theta\left(a_{m}+s_{0}-1\right)$ with $\theta \in \mathcal{V}_{r 1}\left(s_{0}-2-r\right)$, and $\pi^{(m+1)} \in \mathcal{V}_{r 1}\left(n-a_{m+1}-s_{0}+1\right)$. In other words, there exist $m$ edges, say $E_{i_{1}}=E_{\pi}, E_{i_{2}}, \ldots, E_{i_{m}}$, such that the linear representation of $\pi$ is either or

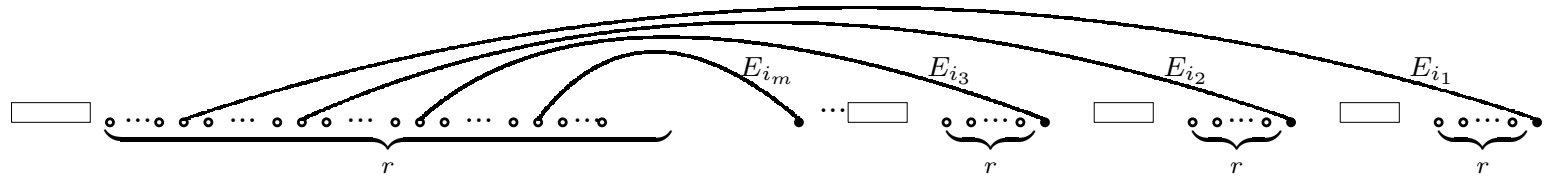

Figure 2. First factorization

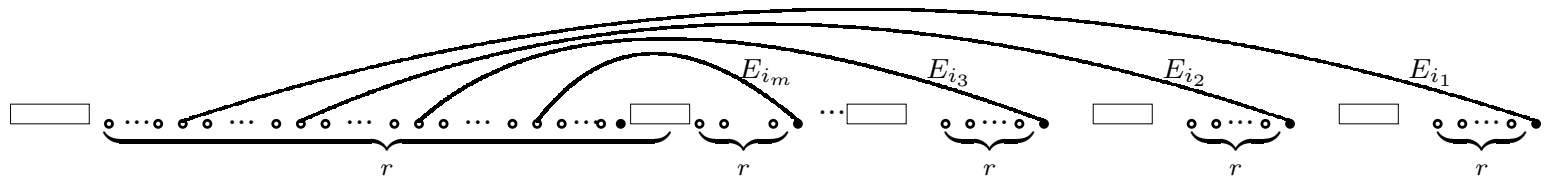

FigurE 3. Second factorization

where each edge $E_{i_{j}}$ covers the edge $E_{i_{j+1}}$, such that the end points of $E_{i_{1}}, \ldots, E_{i_{m}}$ are white vertices, $v_{1}, \ldots, v_{m}$, and there is no black vertex between $v_{i}$ and $v_{j}$, for any $i$ and $j$. 
A $k$-ary tree is a directed tree in which each vertex has degree 0 or $k$ (see, e.g., [32]). The number of $k$-ary trees with $n$ vertices is counted by the $k$-ary numbers, defined by $\frac{1}{k n+1}\left(\begin{array}{c}k n+1 \\ n\end{array}\right)$, for any positive integers $k$ and $n$. Let $\mathcal{T}_{r, n}$ be the set of $r$-ary tree with $n$ nodes. We denote by $T^{1}, \ldots, T^{r}$ the children of its root (from right to left). Now we are ready to define a bijection $\Phi$ recursively. Firstly, the empty contraction maps to the empty $(r+1)$-ary tree, which gives the bijection $\Phi: \mathcal{V}_{r 1}(0) \mapsto \mathcal{T}_{r+1,0}$. Define $F_{\pi}^{\prime}=\left\{k_{1}, k_{2}, \ldots, k_{m}\right\}$ to be an ordered subset (that is, $k_{1}>k_{2}>\ldots>k_{m}$ ) of $F_{\pi}$, such that the node $k_{i}$ is the end point of the edge $E_{i_{m+1-i}}$. Define the minimal vertex of $F_{\pi}$ by $k_{0}$, that is, $k_{0}=\min _{i \in F_{\pi}} i$. Suppose we have defined the bijection $\Phi: \mathcal{V}_{r 1}(m) \mapsto \mathcal{T}_{r+1, m}$ for all $m<n$. For $\pi \in \mathcal{V}_{r 1}(n)$, according to the factorizations of the contraction $\pi$ as described in Lemma 10, there are two cases, for all $m \geq 1$ :

- If $\pi^{(m)}=\emptyset$ (see Figure 2) then we define the $\left(k_{m}-k_{0}+1\right)$-th child of $T$ to be $T^{k_{1}-k_{0}+1}=$ $\Phi\left(\pi^{(m+1)}\right)$, and the $\left(k_{i}-k_{0}+1\right)$-th child of $T$ to be $\left.T^{k_{i}-k_{0}+1}=\Phi^{(} \pi^{(i-1)}\right)$, for each $i=$ $2,3, \ldots, m$.

- If $\pi^{(m)} \neq \emptyset$ (see Figure 3) then define the $(r+1)$-th child of $T$ to be $T^{r+1}=\Phi\left(\pi^{(m+1)}\right)$, and the $\left(k_{i}-k_{0}+1\right)$-st child of $T$ to be $\left.T^{k_{i}-k_{0}+1}=\Phi^{(} \pi^{(i)}\right)$, for each $i=1,2,3, \ldots, m$.

In the case of $m=0$, we define our tree $T$ to be a $(r+1)$-ary tree with root having only one child, which is $T^{r+1}=\Phi\left(\pi^{(1)}\right)$. We can see that if there is no child $T^{r+1}$ of the root of the $(r+1)$-ary tree $T$, then this tree corresponds to a noncrossing contraction with a factorization as described in Figure 2] otherwise, the tree corresponds to a noncrossing contraction with a factorization as described in Figure 3. By induction on the length of the noncrossing contractions and the unique construction of the map $\Phi$, we have that $\Phi$ is invertible. It follows that is a bijection between the set of the noncrossing contractions $\mathcal{V}_{r 1}(n)$ and the set $\mathcal{T}_{r+1, n}$ of $(r+1)$-ary trees. Thus, we have shown the following result.

Theorem 11. There is a bijection between the set of noncrossing contractions in $\mathcal{V}_{r 1}(n)$ and the set $\mathcal{T}_{r+1, n}$ of $(r+1)$-ary trees.

Let $G_{r}(x, y)$ be the generating function for the number of noncrossing contractions in $\mathcal{V}_{r 1}(n)$ with exactly $m$ arcs, that is,

$$
G_{r}(x, y)=\sum_{n \geq 0} \sum_{\pi \in \mathcal{V}_{r 1}(n)} x^{n} y^{\# \operatorname{arcs} \text { in } \pi} .
$$

Then the bijection $\phi$ gives that $G_{r}(x, y)=\left(1+x G_{r}(x, y)\right)\left(1+x y G_{r}(x, y)\right)^{r}$. By Lagrange inversion formula [36] on the above equation, we obtain that

$$
G_{r}(x, y)=\sum_{n \geq 1} \frac{x^{n-1}}{n} \sum_{i=0}^{n-1}\left(\begin{array}{l}
n \\
i
\end{array}\right)\left(\begin{array}{c}
r n \\
n-1-i
\end{array}\right) y^{n-1-i}
$$

As a consequence, we have the following theorem.

Theorem 12. The noncrossing normally ordered from of $\left(a^{r} a^{\dagger}\right)^{n}$ is given by

$$
\mathcal{N C}\left(\left(a^{r} a^{\dagger}\right)^{n}\right)=\sum_{j=0}^{n} \frac{1}{n+1}\left(\begin{array}{c}
n+1 \\
j+1
\end{array}\right)\left(\begin{array}{c}
r n+r \\
j
\end{array}\right)\left(a^{\dagger}\right)^{n-j} a^{r n-j}
$$

Using similar arguments as in the construction of the bijection $\Phi$, one can obtain a bijection between the set of the noncrossing contractions of $\left(a\left(a^{\dagger}\right)^{r}\right)^{n}$ and the set of $(r+1)$-ary trees with $n$ nodes.

Theorem 13. The noncrossing normally ordered from of $\left(a\left(a^{\dagger}\right)^{r}\right)^{n}$ is given by

$$
\mathcal{N C}\left(\left(a\left(a^{\dagger}\right)^{r}\right)^{n}\right)=\sum_{j=0}^{n} \frac{1}{n+1}\left(\begin{array}{c}
n+1 \\
j+1
\end{array}\right)\left(\begin{array}{c}
r n+r \\
j
\end{array}\right)\left(a^{\dagger}\right)^{r n-j} a^{n-j}
$$


5.2. The case $\left(a+\left(a^{\dagger}\right)^{2}\right)^{n}$ and lattice paths. In this section, we present a bijection $\Phi$ between the set of contractions $\mathcal{W}_{12}(n)$ of the word monomials in the expression $F\left(a, a^{\dagger}\right)=\left(a+\left(a^{\dagger}\right)^{2}\right)^{n}$ and a special set $\mathcal{P}_{n}$ of $L$-lattice paths. These are lattice paths on $\mathbb{Z}^{2}$, which are of length $4 n$ and go from $(0,0)$ to $(3 n, 0)$. Moreover, the paths never go below the $x$-axis with the steps $H=(2,1), D=(1,-1)$ and $L=(1,2)$, and with no three consecutive $D$ steps (that is, there is no triple $D D D$ ). The paths of length six are described in Figure 4
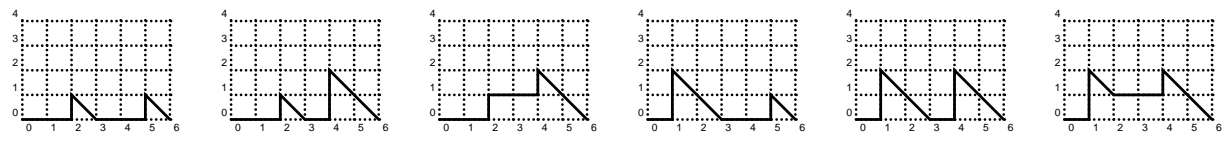

Figure 4. The set $\mathcal{P}_{2}$ of lattices paths

Let $P$ be a path in $\mathcal{P}_{n}$. Then, using a first return decomposition (first return to $x$-axis) we obtain the factorization of $P$ as either

$$
H D P^{\prime}, L D D P^{\prime}, L D P^{\prime} H D D P^{\prime \prime}, H P^{\prime} H D D P^{\prime \prime} D \text {, or } L D P^{\prime} L D P^{\prime \prime} H D D H D D P^{\prime \prime \prime} \text {, }
$$

where $P^{\prime}, P^{\prime \prime}, P^{\prime \prime \prime}$ are paths of smaller length (see Figure 5). On the basis of this observation, the bijection $\Phi$ can be defined recursively. Firstly, the empty contraction maps to the empty path, which gives the bijection $\Phi: \mathcal{W}_{12}(0) \mapsto \mathcal{P}_{0}$. Suppose we have defined the bijection $\Phi: \mathcal{W}_{12}(m) \mapsto \mathcal{P}_{m}$ for all $m<n$. For $\pi \in \mathcal{W}_{12}(n)$, according to the factorizations of the contraction $\pi$, there are five cases:

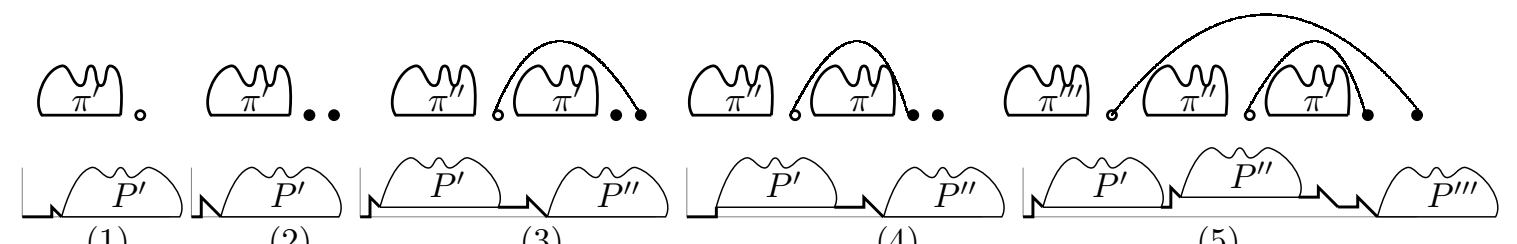

(1)

(2)

(3)

(4)

(5)

Figure 5. The bijection $\Phi$.

(i) The contraction $\pi$ starts with a white vertex, namely $\pi=1^{\prime} \pi^{\prime} \in \mathcal{W}_{12}(n)$. We define $\Phi(\pi)$ to be the joint of the steps $H D$ and the path $P^{\prime}=\Phi(\beta)$, where $\beta_{i}=\pi_{i}^{\prime}-1$ (define $i^{\prime}-d=(i-d)^{\prime}$ ), for each $i=1,2, \ldots, n-1$ (see Figure $5(1)$ ).

(ii) The contraction $\pi$ starts with a black vertex, namely $\pi=1 \pi^{\prime} \in \mathcal{W}_{12}(n)$. We define $\Phi(\pi)$ to be the joint of the steps $L D D$ and the path $P^{\prime}=\Phi(\beta)$, where $\beta_{i}=\pi_{i}^{\prime}-1$, for each $i=1,2, \ldots, n-1$ (see Figure $5(2)$ ).

(iii) The contraction $\pi$ starts with an arc followed by a black vertex with degree zero, that is, $\pi=12 \pi^{\prime} 1 \pi^{\prime \prime} \in \mathcal{W}_{12}(n)$, where in $\pi^{\prime}$ does not occur the letter 2 . We define $\Phi(\pi)$ to be the joint of the step $L D$, the path $P^{\prime}=\Phi\left(\beta^{\prime}\right)$, the steps $H D D$, and the path $P^{\prime \prime}=\Phi\left(\beta^{\prime \prime}\right)$, where, for each $i, \beta_{i}^{\prime}=\pi_{i}^{\prime}-2$ and $\beta_{i}^{\prime \prime}=\pi_{i}^{\prime \prime}-\max (2, \ell)$ such that $\ell$ is the maximal letter of $\pi^{\prime}$ (see Figure $5(3))$.

(iv) The contraction $\pi$ starts with a black vertex of degree zero followed by an arc, that is, $\pi=$ $12 \pi^{\prime} 2 \pi^{\prime \prime} \in \mathcal{W}_{12}(n)$, where in $\pi^{\prime \prime}$ does not occur the letter 1 . We define $\Phi(\pi)$ to be the joint of the step $H$, the path $P^{\prime}=\Phi\left(\beta^{\prime}\right)$, the steps $H D D$, and the path $P^{\prime \prime}=\Phi\left(\beta^{\prime \prime}\right)$, where, for each $i, \beta_{i}^{\prime}=\pi_{i}^{\prime}-2$ and $\beta_{i}^{\prime \prime}=\pi_{i}^{\prime \prime}-\max (2, \ell)$ such that $\ell$ is the maximal letter of $\pi^{\prime}$ (see Figure $5(3)$ ).

(v) The contraction $\pi$ starts with two arcs, that is $\pi=12 \pi^{\prime} 1 \pi^{\prime \prime} 2 \pi^{\prime \prime \prime} \in \mathcal{W}_{12}(n)$. We define $\Phi(\pi)$ to be the joint of the steps $L D$, the path $P^{\prime}=\Phi\left(\beta^{\prime}\right)$, the steps $L D$, the path $P^{\prime \prime}=\Phi\left(\beta^{\prime \prime}\right)$, the steps $H D D H D D$, and the path $P^{\prime \prime \prime}=\Phi\left(\beta^{\prime \prime \prime}\right)$; for each $i, \beta_{i}^{\prime}=\pi_{i}^{\prime}-2, \beta_{i}^{\prime \prime}=\pi_{i}^{\prime \prime}-\max \left(2, \ell^{\prime}\right)$ 
and $\beta^{\prime \prime \prime}=\pi_{i}^{\prime \prime \prime}-\max \left(2, \ell^{\prime}, \ell^{\prime \prime}\right)$, such that $\ell^{\prime}$ and $\ell^{\prime \prime}$ are the maximal letters of $\pi^{\prime}$ and $\pi^{\prime \prime}$, respectively (see Figure 5(4)).

The inverse map of $\Phi$ is clearly understood from the above cases (see the factorizations of the paths in $\mathcal{P}_{n}$ and the factorizations of the contractions in $\mathcal{W}_{12}(n)$ as described in Figure 5 ).

Theorem 14. The map $\Phi$ is a bijection between the set of contractions in $\mathcal{W}_{12}(n)$ and the set of lattices paths in $\mathcal{P}_{n}$. Moreover, for any contraction $\pi \in \mathcal{W}_{12}(n)$, we have:

(i) the number of arcs in the linear representation of $\pi$ equals the number of HDD in the corresponding path $\Phi(\pi)$;

(ii) the number of white vertices in the linear representation of $\pi$ equals the number of $H D$ in the corresponding path $\Phi(\pi)$.

Let $P(x, y)=\sum_{n \geq 0} \sum_{P \in \mathcal{P}_{n}} x^{n} y^{H D D(P)}$ be the generating function for the number of paths $P$ of length $3 n$ in $\mathcal{P}_{n}$, respect to the semilength $n$ of $P$ and the number of occurrences $H D D(P)$ in the string $H D D$ in $P$. Then by the factorization of the paths in $\mathcal{P}$, see Figure 5 , we obtain that the generating function $P(x, y)$ satisfies

$$
P(x, y)=1+2 x P(x, y)+2 x^{2} y P^{2}(x, y)+x^{3} y^{2} P^{3}(x, y)
$$

From (77) we obtain that $P(x, y)=A_{1, s}(x, y, 1)$. Thus we can state the following result.

Corollary 15. The noncrossing normally ordered form of $\left(a+\left(a^{\dagger}\right)^{2}\right)^{n}$ is given by

$$
\mathcal{N C}\left(\left(a+\left(a^{\dagger}\right)^{2}\right)^{n}\right)=\sum_{j=0}^{n} \sum_{i=j}^{n} \frac{1}{n+1}\left(\begin{array}{c}
n+1 \\
j+1
\end{array}\right)\left(\begin{array}{c}
n-j \\
n-i
\end{array}\right)\left(\begin{array}{c}
2 n-2 i \\
j
\end{array}\right)\left(a^{\dagger}\right)^{2 n-2 i-j} a^{i-j}
$$

5.3. $\left(a+a^{\dagger}\right)^{n},\left(a a^{\dagger}\right)$, and 2-Motzkin paths. A 2-Motzkin path of length $n$ is a path on the plane from the origin $(0,0)$ to $(n, 0)$ consisting

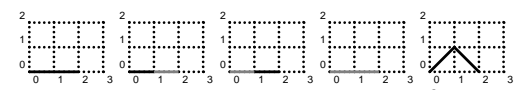

FiguRE 6. 2-Motzkin paths of length 2.

of up steps, down steps, level steps colored black, and level steps colored gray, such that the path does not go below the $x$-axis. We will use $U, D, L$, and $L^{\prime}$, to represent the up, down, black level, gray level steps, respectively (see Figure 6). Most probably, the notion of 2-Motzkin path firstly appeared in the work of Delest and Viennot [13] and has been studied in a number of works, including [2, 14]. First, we describe a bijection $\Psi$ between the set of linear representations $\mathcal{V}_{11}(n)$ and the set $\mathcal{M}_{n}$ of 2-Motzkin paths of length $n$. Let $\pi=\pi_{2 n} \pi_{2 n-1} \ldots \pi_{1}$ be a linear representation in $\mathcal{V}_{11}(n)$. We read each time two vertices from $\pi$ from right to left and successively generate the 2-Motzkin path. When a black vertex $\pi_{2 j-1}$ with degree zero is followed by a white vertex $\pi_{2 j}$ with degree zero, then in the 2 -Motzkin path we add a black level step $L$. When a black vertex $\pi_{2 j-1}$ with degree one is followed by a white vertex $\pi_{2 j}$ with degree one, then in the 2-Motzkin path we add a gray level step $L^{\prime}$. When a black vertex $\pi_{2 j-1}$ with degree one (resp. zero) is followed by a white vertex $\pi_{2 j}$ with degree zero (resp. one), then in the 2-Motzkin path we add an up (resp. down) step $U$ (resp. D). The reverse of the map $\Psi$ is obvious.

Proposition 16. There is a bijection $\Psi$ between the set of noncrossing contractions in $\mathcal{V}_{11}(n)$ and the set of 2-Motzkin paths of length $n$. Moreover, $\pi \in \mathcal{V}_{11}(n)$ has exactly $m$ edges if and only if the number of black level steps and up-steps in $\Psi(\pi)$ equals $m$. 
The above proposition gives that the noncrossing normally ordered form of $\left(a a^{\dagger}\right)^{n}$ is given by

$$
\mathcal{N C}\left(\left(a a^{\dagger}\right)^{n}\right)=\sum_{j=0}^{n} \frac{1}{n+1}\left(\begin{array}{c}
n+1 \\
j+1
\end{array}\right)\left(\begin{array}{c}
n+1 \\
j
\end{array}\right)\left(a^{\dagger}\right)^{n-j} a^{n-j}
$$

(see Theorem 13) where the numbers $\frac{1}{n+1}\left(\begin{array}{c}n+1 \\ j+1\end{array}\right)\left(\begin{array}{c}n+1 \\ j\end{array}\right)$ are the so-called Narayana numbers (see, e.g., [37]).

Now, we describe a second bijection $\Theta$ between the set of linear representations $\mathcal{W}_{11}(n)$ and the set $\mathcal{M}_{n}$ of 2-Motzkin paths of length $n$. Let $\pi=\pi_{2 n} \pi_{2 n-1} \ldots \pi_{1}$ be a linear representation in $\mathcal{W}_{11}(n)$. We read $\pi$ from right to left to generate the 2-Motzkin path. When a black (resp. white) vertex $\pi_{j}$ with degree zero is read, then in the 2-Motzkin path we add a black (resp. gray) level step $L$ (resp. $\left.L^{\prime}\right)$. When a black (resp. white) vertex with degree one is read, then in the 2-Motzkin path we add an up (resp. down) step $U$ (resp. D). The reverse of the map $\Theta$ is again obvious.

Proposition 17. There is a bijection $\Theta$, between the set of noncrossing contractions in $\mathcal{W}_{11}(n)$ and the set of 2-Motzkin paths of length $n$. Moreover, $\pi \in \mathcal{W}_{11}(n)$ has exactly $m$ edges and $d$ white vertices if and only if the number of up-steps in $\Theta(\pi)$ equals $m$ and the number of gray level steps in $\Theta(\pi)$ is $d-m$.

The above proposition gives that the noncrossing normally ordered form of $\left(a+a^{\dagger}\right)^{n}$ is given by

$$
\mathcal{N C}\left(\left(a+a^{\dagger}\right)^{n}\right)=\sum_{j=0}^{n} \sum_{i=j}^{n-j} c_{j}\left(\begin{array}{c}
n \\
i+j
\end{array}\right)\left(\begin{array}{c}
i+j \\
2 j
\end{array}\right)\left(a^{\dagger}\right)^{n-i-j} a^{i-j}
$$

as described in Theorem 9

\section{Open PROBlems}

A number of directions for further research seem to arise naturally. The first and foremost problem consists in deriving a more physical understanding of the process of noncrossing normal ordering. As discussed in Section 2, the result of noncrossing normal ordering cannot be reproduced by the conventional normal ordering where some kind of commutation relations is assumed. Thus, the statistics resulting from the noncrossing normal ordering is a new and nontrivial phenomenon which clearly deserves closer study. In this context it is not even clear whether one should call the operators for which the noncrossing normal ordering is applied "bosonic" anymore.

Let us also state two mathematical problems:

- Study the nonnesting normally ordered form of a given expression $F\left(a, a^{\dagger}\right)$. We say that the two edges $e=(a, b)$ and $e^{\prime}=(c, d)$ are nesting if $a<c<d<b$ (that is, the edge $e$ covers the edge $e^{\prime}$ ) or $c<a<b<d$ (that is, the edge $e^{\prime}$ covers the edge $e$ ). In the case of nonnesting partitions, see [24].

- Study the distribution of a given statistic on the set of normally ordered form of a given expression. For example, study the asymptotic behavior of the number of edges that cover other edges in the normally ordered form of $\left(a\left(a^{\dagger}\right)^{r}\right)^{n}$, when $n$ tends to infinity.

Acknowledgments. The authors would like to thank Ed Corrigan, Chris Fewster, Gherardo Piacitelli, and Tony Sudbery for helpful discussion; Paweł Błasiak and Jacob Katriel for encouragement and interest in this work. 


\section{REFERENCES}

[1] M. Anshelevich, Documenta Math. 6 (2001) 343.

[2] E. Barcucci, A. Del Lungo, E. Pergola and R. Pinzani, Lecture Notes in Computer Science 959:254-263, Springer, Berlin, 1995.

[3] J. D. Bjorken and S. D. Drell, Relativistic quantum fields, McGraw-Hill Book Co., New York, 1965

[4] P. Błasiak, Combinatorics of boson normal ordering and some applications, PhD Thesis, Institute of Nuclear Physics PAS: Kraków and Université P. et M. Curie: Paris, 2005. quant-ph/0507206

[5] P. Błasiak, K. A. Penson, and A. I. Solomon, Phys. Lett. A 309 (2003) 198.

[6] P. Błasiak, K. A. Penson, and A. I. Solomon, Ann. Comb. 7 (2003) 127.

[7] P. Błasiak, K.A. Penson and A.I. Solomon, Lett. Math. Phys. 67:13-23, 2004.

[8] P. Błasiak, A. Horzela, K. A. Penson, G. H. E. Duchamp and A. I. Solomon, Phys. Lett. A 338 (2005) 108.

[9] P. Błasiak, K. A. Penson, A. I. Solomon, A. Horzela and G. H. E. Duchamp, J.Math.Phys. 46 (2005) 052110.

[10] M. Bozeko, B. Kümmerer and R. Speicher, Comm. Math. Phys. 185 (1997) 129.

[11] K. E. Cahill and R. J. Glauber, Phys. Rev. 177 (1969) 1857.

[12] W.Y.C. Chen, Eva Y.P. Deng and Rosena R.X. Du, Europ. J. Combin. 26(2): 237-243, 2005.

[13] M. Delest and G. Viennot, Theoret. Comput. Sci. 34:169-206, 1984.

[14] E. Deutsch and L.W. Shapiro, Discrete Math. 256:655-670, 2002.

[15] E. G. Effros and M. Popa, Proc. Natl. Acad. Sci. USA 100 (2003) 8629.

[16] R. J. Glauber, Phys. Rev. 130 (1963) 2529.

[17] J. Katriel, Lett. Nuovo Cimento 10(13):565-567, 1974.

[18] J. Katriel, J. Phys. A 16(17):4171-4173, 1983.

[19] J. Katriel, Phys. Lett. A 273(3):159-161, 2000.

[20] J. Katriel, J. Opt. B: Quantum Semiclass. Opt. 4:S200-S203, 2002.

[21] J. R. Klauder, J. Math. Phys. 4 (1963) 1055.

[22] J. R. Klauder and B.-S. Skagerstam, Coherent states. Applications in physics and mathematical physics, World Scientific Publishing Co., Singapore, 1985.

[23] J. R. Klauder and E. C. G. Sudarshan, Fundamentals of quantum optics, W. A. Benjamin, Inc., New York, 1968

[24] M. Klazar, Europ. J. Combin. 17:53-68, 1996.

[25] M. Klazar, Adv. Appl. Math. 30:126-136, 2003.

[26] J. McCammond, American Mathematical Monthly 113 (2006), 598-610.

[27] T. Mansour, M. Schork and S. Severini, Phys. Lett. A 362:214-220, 2007.

[28] M. d'Ocagne, Amer. J. Math. 9:353-380, 1887.

[29] R. Simion, Discr. Math. 217, 367-409, 2000.

[30] N.J.A. Sloane, The On-Line Encyclopedia of Integer Sequences, www.research.att.com/ ${ }^{\sim}$ njas/sequences.

[31] E. C. G. Sudarshan, Phys. Rev. Lett. 10 (1963) 277.

[32] R. Stanley, Enumerative Combinatorics, vol. 1, Wadsworth and Brooks/Cole, Pacific Grove, CA, 1986.

[33] A. Varvak, J. Combin. Theory Ser. A 112(2):292-307, 2005.

[34] W.C. Yang, Discr. Math. 156:247-252, 1996.

[35] G. C. Wick, Physical Rev. (2) 80, (1950), 268.

[36] H. Wilf, Generatingfunctionology, Academic Press, New York, 1990.

[37] D. Zeilberger, Intern. J. Computer Math 29:201-215, 1989. 